\title{
La métamorphose des amphibiens : un modèle prometteur pour étudier les protéases de la matrice
}

\author{
La métamorphose des amphibiens offre un modèle \\ intéressant d'étude du développement post-embryonnaire, \\ et en particulier des cascades de contrôle morphogénétique \\ mises en place par les hormones thyrö̈diennes. Ces \\ hormones entraînent chez le xénope l'activation de gènes \\ codant pour des métalloprotéases qui dégradent la matrice \\ extracellulaire. Celles-ci semblent jouer un rôle important
}

\author{
dans le remodelage des tissus et leur régression au cours \\ de la métamorphose. L'étude de leur mode d'action, \\ et du contrôle de leur expression et de leur activité \\ par les hormones thyrö̈diennes dans ce modèle, \\ pourrait apporter des informations utiles \\ à la compréhension de l'action de ces enzymes \\ chez les mammiferes.
}

$\mathbf{P}$ ar son côté spectaculaire et hautement organisé, la métamorphose des amphibiens a attiré très tôt de nombreux chercheurs qui souhaitaient disposer d'un modèle de développement post-embryonnaire. Il s'agit d'un phénomène complexe faisant intervenir des processus cellulaires et tissulaires aussi différents que la différenciation, l'apoptose ou la morphogenèse. Les événements les mieux caractérisés chez les amphibiens, comme le xénope (Xenopus laevis), sont la perte de la queue, le remodelage de l'intestin et la morphogenèse des membres. Un des aspects les plus fascinants de ce processus est qu'un seul signal hormonal, celui délivré par les hormones thyroïdiennes, est capable de provoquer des effets opposés sur différents tissus de l'organisme. La métamorphose des amphibiens est donc devenue, au même titre que celle des insectes, un modèle d'étude des cascades de contrôle génique mises en route par l'action d'une hormone.

Les hormones thyroïdiennes, notamment la T3 (3,5,3' triiodo-L-thyronine) qui est la forme biologiquement active, agissent via deux récepteurs appelés $\operatorname{TR} \alpha$ et $\operatorname{TR} \beta$, des membres de la superfamille des récepteurs nucléaires d'hormones. Ceux-ci peuvent former des hétérodimères avec d'autres récepteurs appe- lés RXR (retinoid X receptor) ( $\mathrm{m} / \mathrm{s} 1998$, $\left.n^{\circ} 11, p .1211\right)$. Ces hétérodimères se fixent sur des éléments de réponses aux hormones thyroïdiennes, les TRE (pour thyroid hormone response ele$m e n t$ ), situés dans les promoteurs des gènes cibles. En présence d'hormones, les hétérodimères TR-RXR activent la transcription de leurs gènes cibles alors qu'en leur absence, ils la répriment.

\section{Une cascade d'expression génique contrôlée par les hormones thyroïdiennes}

Afin de comprendre les événements observés pendant la métamorphose du xénope, l'équipe de Don Brown à Baltimore a cherché à identifier le programme d'expression génique qui est sous le contrôle des hormones thyroïdiennes. Ainsi, plusieurs études de criblages différentiels ont été réalisées à partir de différents organes et ont permis de déterminer trois classes majeures de gènes dont certains ont été identifiés [1,2] (figure 1). La première classe est constituée d'un petit nombre de gènes qui sont réprimés par les hormones thyroïdiennes et qui codent pour des protéines membranaires ou extracellulaires. Les deux autres classes comportent des gènes qui sont tous activés par les hormones thyroïdiennes, mais se dis- tinguent par le caractère précoce ou plus tardif de cette activation. Les gènes activés de façon précoce codent essentiellement pour des facteurs de transcription, dont TR $\beta$, et une métalloprotéase de la matrice (MMP-matrix metalloprotease), la stromélysine 3. Parmi les gènes activés de façon tardive, certains codent pour des protéines de la matrice extracellulaire comme la fibronectine, l'intégrine $\alpha 1$, et d'autres pour des protéines de modification de la matrice extracellulaire comme la collagénase 3, une autre MMP.

Cette cascade de contrôle de l'expression génique n'est pas encore comprise en détail, notamment en ce qui concerne les relations précises entre les différents acteurs. Elle est néanmoins très intéressante à mettre en perspective avec ce que l'on sait du rôle des hormones thyroïdiennes chez les mammifères. En effet, chez ces organismes, elles contrôlent de nombreux paramètres physiologiques comme le métabolisme énergétique, la fonction cardiaque ou la thermogenèse. Cette multiplicité d'actions a constitué un obstacle important pour notre compréhension de leur mode d'action précis. La mise en évidence d'une cascade de contrôle relativement simple dans son principe chez le xénope pourrait permettre de disposer d'un modèle 


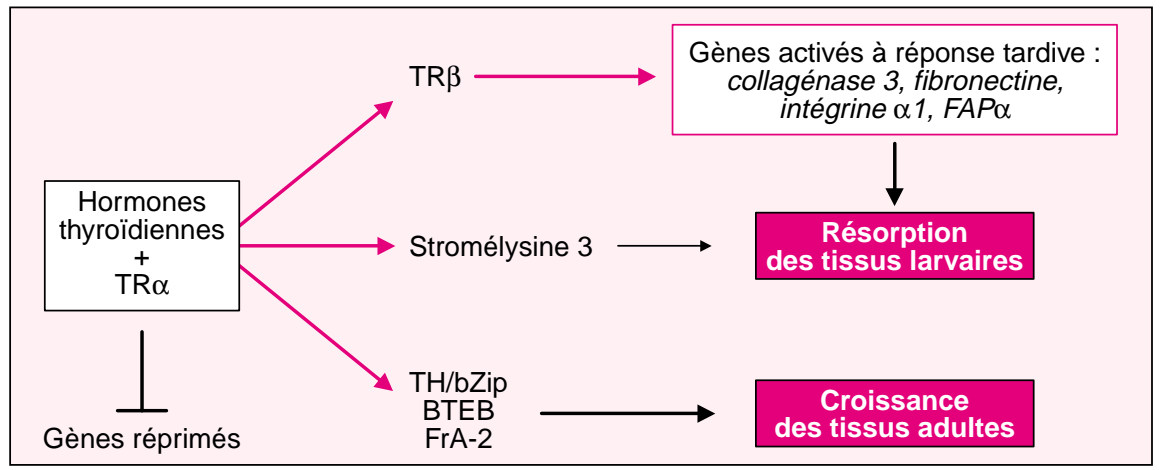

Figure 1. Hypothèse de cascade de contrôle génique par les hormones thyroïdiennes dans la queue du xénope. L'hormone se fixerait tout d'abord au récepteur TR $\alpha$, qui est présent de manière abondante avant le début de la métamorphose. Ceci entraînerait l'induction de gènes précoces laprès 2 à 4 heures), dont le récepteur TR $\beta$. Ce dernier serait responsable de l'activation, en présence de l'hormone, des gènes dits tardifs (induction après 12 à 24 heures). Les gènes réprimés au cours de la métamorphose n'ont pas d'homologues connus, et leur fonction reste encore mystérieuse. TH/bZip: thyroid hormone induced bZip gene ; BTEB : basic transcription element binding protein ; FRA-2: Fos related antigen 2 ; FAP $\alpha$ : fibroblast activation protein $\alpha$.

d'action moléculaire de ces hormones chez les mammifères. Cela est d'autant plus vrai que les phénotypes des souris pour lesquelles les gènes $\operatorname{TR} \alpha$ et/ou TR $\beta$ ont été invalidés montrent que ces hormones ont un rôle crucial dans le développement post-embryonnaire, notamment au moment du sevrage. Par exemple, chez les souris $T R \alpha^{-1}$, on observe un défaut de maturation de l'intestin grêle au moment du sevrage, et un retard de la maturation osseuse [3]. De là à penser que ces hormones contrôlent le développement postembryonnaire chez de nombreux vertébrés via des mécanismes globalement similaires, il n'y a qu'un pas... qu'il ne faut tout de même pas franchir trop légèrement ! Il existe en effet des différences importantes entre les deux systèmes. Par exemple, chez les amphibiens, l'expression de $\operatorname{TR} \beta$ est sous le contrôle direct des hormones thyroïdiennes, et ce rétrocontrôle positif, qui n'existe pas chez les mammifères, joue un rôle majeur dans le déclenchement de la métamorphose. Il n'en reste pas moins que l'étude comparative au niveau moléculaire de la réponse aux hormones thyroïdiennes au cours du développement post-embryonnaire des amphibiens et des mammifères massive des cellules épithéliales larvaires et à une prolifération importante des cellules souches pour former le tissu épithélial adulte [4] (figure 2). Au cours de la régression de la queue, deux populations de fibroblastes migrent vers les lamelles de collagène voisines, entraînant leur destruction puis l'apoptose des cellules musculaires et épidermiques de la queue [5] (figure 3). Ces deux exemples montrent que différentes matrices extracellulaires sont modifiées lors de la métamorphose, ce qui fournit un premier indice sur l'implication possible des MMP dans ce processus. Le deuxième argument fort est l'observation, par Don Brown, d'un contrôle de l'expression des MMP au cours de la métamorphose $[1,2]$.

Les MMP sont des enzymes extracellulaires dont l'activité est dépendante $\mathrm{du} \mathrm{Zn}^{2+}$ et qui sont capables de dégrader les composants protéiques de la matrice extracellulaire. Parmi elles on trouve les collagénases, les stromélysines et les gélatinases, dont les substrats sont différents mais chevauchants. Ces protéines sont généralement sécrétées sous une forme inactive, appelée pro-enzyme, puis activées dans le milieu extracellulaire par clivage protéolytique du pro-peptide (figure 4). Seules la stromélysine 3 et les MMP liées à la membrane sont activées de manière intracellulaire par la furine.

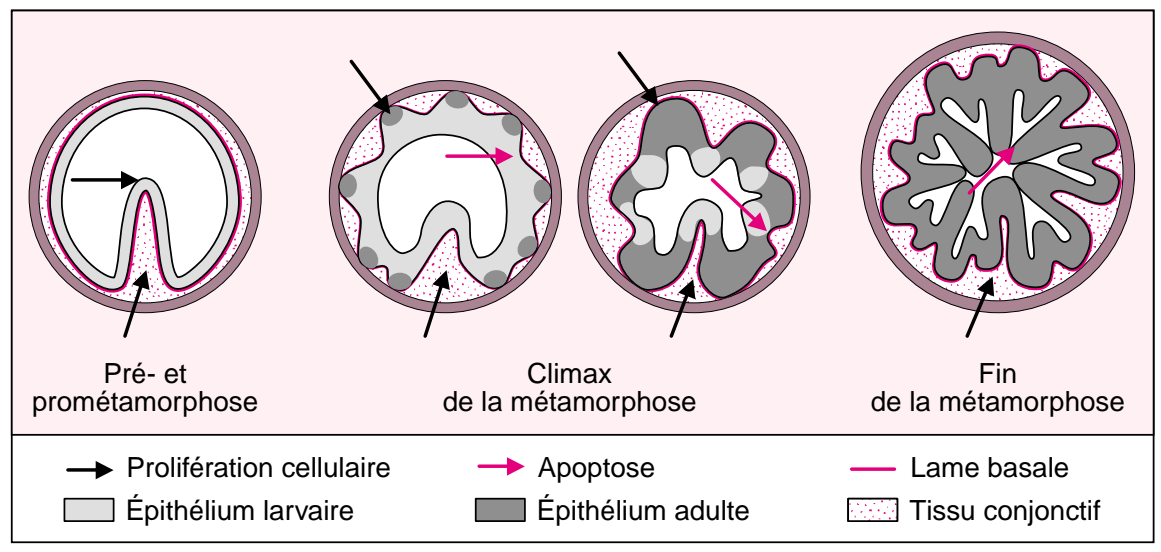

Figure 2. Métamorphose de l'intestin. Au cours de la métamorphose de I'intestin, le tissu conjonctif prolifère tandis que l'épithélium larvaire entre en apoptose, laissant la place à l'épithélium adulte. Ces modifications sont associées à la formation de replis intestinaux et à des changements importants de l'état de la lame basale. Le climax est la période de la métamorphose où les changements morphologiques sont les plus importants. 


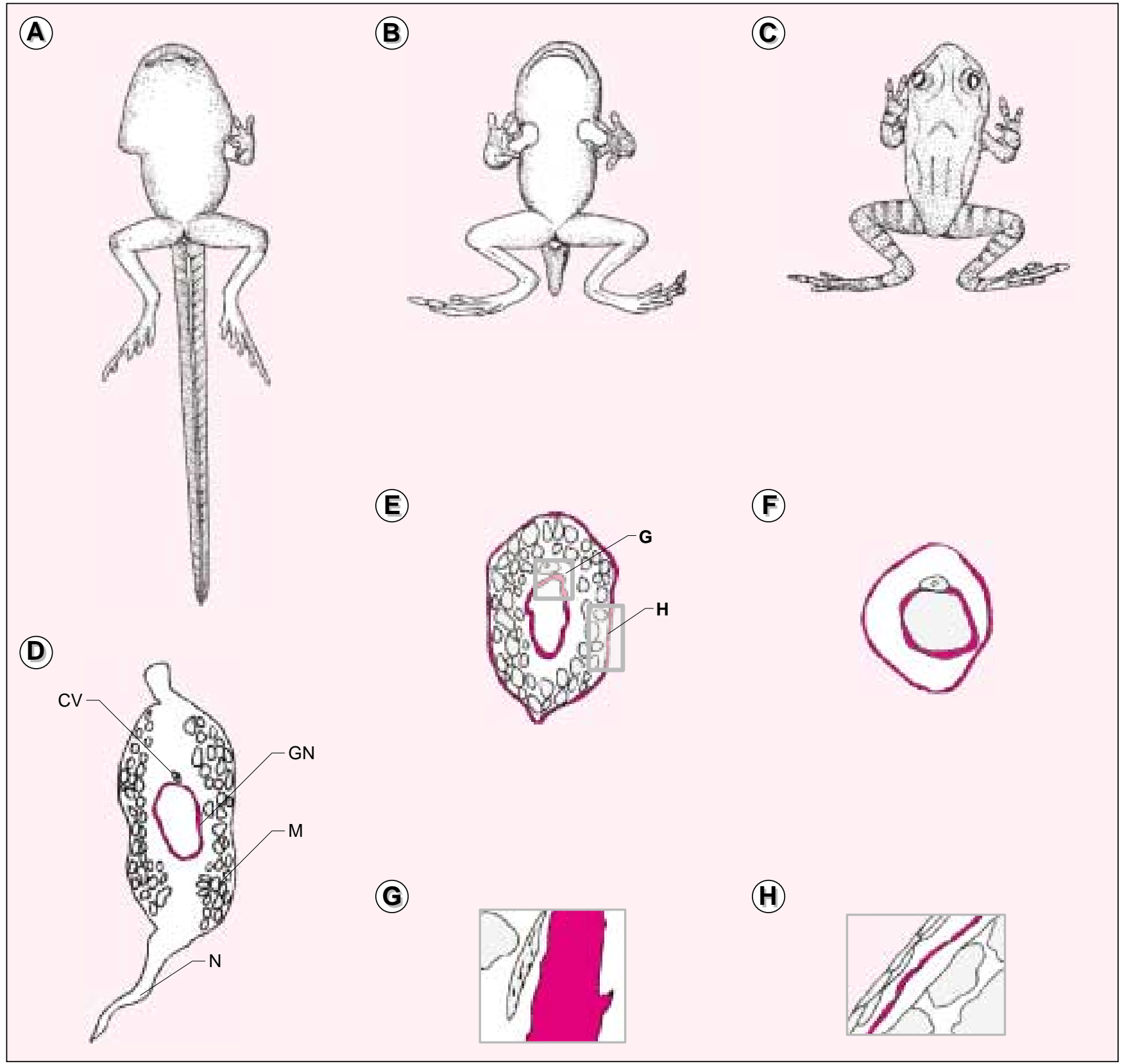

Figure 3. Métamorphose de la queue. A, B, C. Modifications morphologiques du xénope au cours de la métamorphose. D, E et $\boldsymbol{F}$. Coupes transversales, à ces différents stades, de la queue qui régresse progressivement. Sur les schémas $\boldsymbol{G}$ et $\boldsymbol{H}$ sont représentés en rouge les fibroblastes de la gaine de la notochorde (G) et les fibroblastes sousépidermiques $(\mathrm{H})$. Ces deux populations migrent vers les lamelles de collagène qui les entourent au cours de la métamorphose entraînant leur dégradation. CV: colonne vertébrale; GN: gaine de la notochorde, M: muscle; $N$ : nageoire

L'intervention des MMP au cours de la métamorphose des anoures a été suspectée dès 1966 par J. Gross qui a observé une forte augmentation de la dégradation du collagène dans la queue du xénope lors de sa régres$m / s n^{\circ} 11$, vol. 17 , novembre 2001 sion [6]. Depuis, trois gènes codant pour des MMP, et activés par les hormones thyroïdiennes, ont été caractérisés chez le xénope. Il s'agit de la stromélysine 3 (ST3), de la collagénase 3 (Col3) et de la collagénase 4
(Col4). Des études par Northern blot ont permis de montrer que ces 3 gènes sont exprimés dans l'intestin, les membres et la queue, aux moments où les changements s'effectuent dans ces trois organes [7, 8]. 


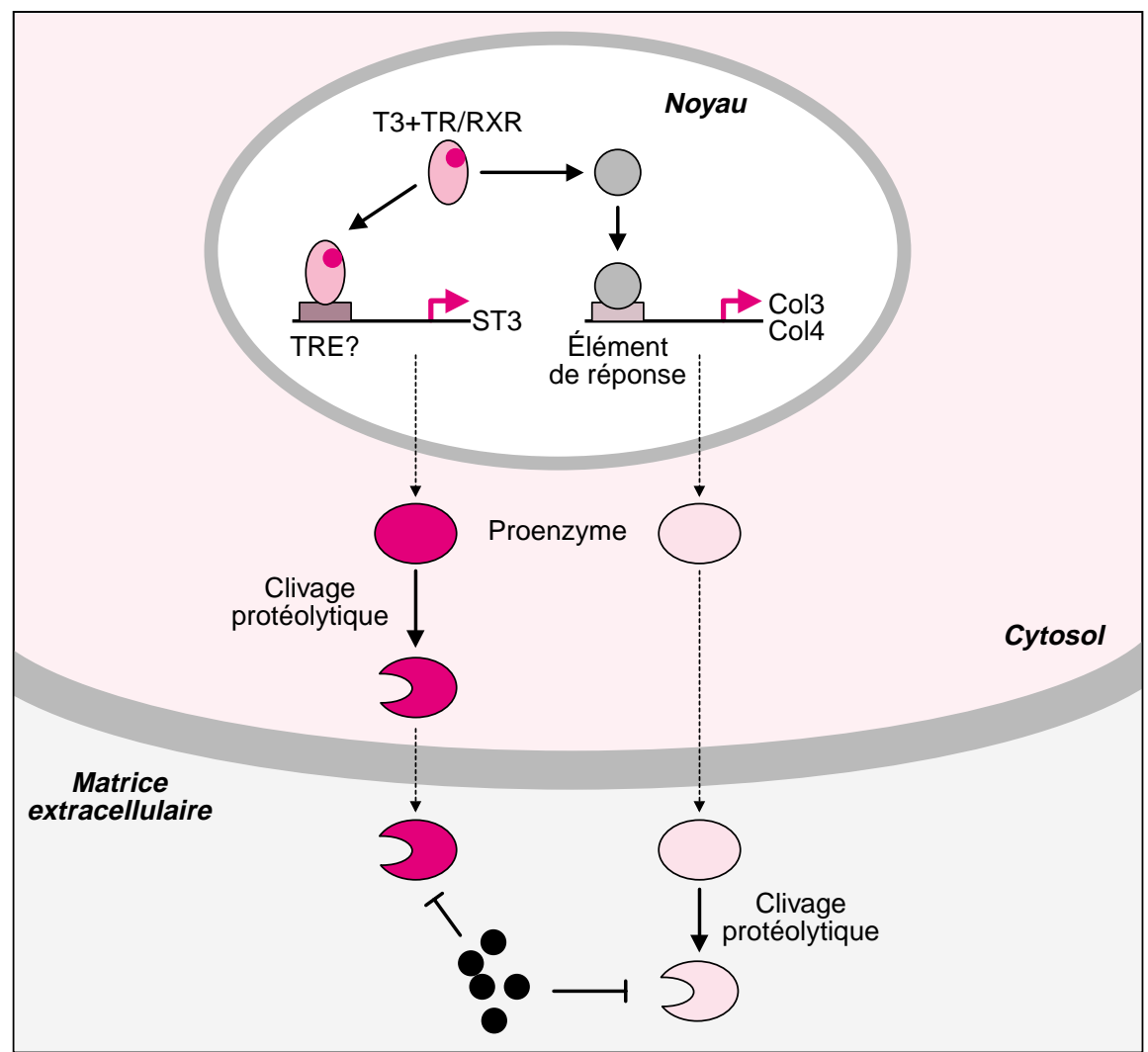

Figure 4. Contrôle de l'expression et de l'activité des métalloprotéases pendant la métamorphose. La fixation de T3 à son récepteur semble entraîner directement une élévation de l'expression du gène codant pour la ST3 (stromélysine), et, indirectement, celle des gènes codant pour la Col3 (collagénase) et la Col4, par l'intermédiaire d'un facteur de transcription $X$ probablement différent pour les deux MMP. La ST3 est activée par clivage protéolytique dans le cytoplasme tandis que Col3 et Col4 sont activées hors des cellules. Dans le milieu extracellulaire, l'activité des MMP peut être modulée par des inhibiteurs spécifiques, les TIMP (Tissue Inhibitors of Metalloproteinases) qui ne sont pas encore connus chez les amphibiens.

En outre, dans ces différents organes, le gène de la ST3 est exprimé de manière plus précoce que ceux de la Col3 et de la Col4

Les expériences d'hybridation in situ ont révélé que pendant la régression de la queue, l'expression des transcrits ST3 et Col3 est augmentée dans les fibroblastes qui envahissent les lamelles de collagènes. Dans les cartilages de la tête, l'expression de la ST3 et de la collagénase 3 est activée, pour la première dans les fibroblastes qui entourent l'épithélium pharobranchial voué à disparaître, et pour la seconde dans toutes les cellules des cartilages qui se résorbent au cours de la métamorphose [9]. Dans

\section{Métalloprotéases}

et hormones thyroïdiennes

Les hormones thyroïdiennes activent l'expression de la ST3 de façon très précoce et ce, en présence de cycloheximide, un inhibiteur de la synthèse protéique [10]. Ceci suggère fortement que les hormones thyroïdiennes activent de façon directe la transcription du gène codant pour la ST3 (figure 4). Cependant, une partie $\mathrm{du}$ promoteur de ce gène (de - $4000 \mathrm{pb}$ à $+600 \mathrm{pb}$ ) a été clonée chez le xénope [11]: il ne semble pas permettre de réponse aux hormones thyroïdiennes, et aucun TRE classique n'a pu être identifié. Ces résultats sont à rapprocher de ceux qui ont été obtenus chez les mammifères, pour lesquels la ST3 a suscité un intérêt particulier, car elle est exprimée de façon excessive dans de nombreux cancers. Les études effectuées sur les promoteurs du gène humain et murin codant pour la ST3 ont permis d'identifier un TRE [12]. Même si les séquences régulatrices ne sont généralement pas conservées entre les espèces, il est tout de même possible d'imaginer que le promoteur de ce gène, chez le xénope, possède effectivement un TRE mais que celui-ci n'a pour l'instant pas encore été localisé.

En ce qui concerne les deux autres MMP, on sait que l'induction de l'expression de la Col3 par les hormones thyroïdiennes est inhibée en présence de cycloheximide [10], ce qui est faveur d'un mécanisme de contrôle indirect. Quant à la Col4, seule la cinétique tardive d'activation par les hormones thyroïdiennes permet de suggérer que ce contrôle est aussi indirect. On peut souligner que, chez l'homme, aucun TRE n'a été caractérisé dans le promoteur de la collagénase 3 , et que la collagénase 4 n'a été décrite pour l'instant que chez le xénope.

Un rôle direct des métalloprotéases dans le remodelage tissulaire ?

L'activité protéolytique des MMP de xénope n'a pour l'instant été déterminée que pour la Col4 [7]. Celle-ci clive différents substrats de la matrice extracellulaire, en particulier le colla- 
gène I. Pour la ST3 et la Col3, les degrés relativement importants d'homologie de séquences avec leurs homologues humains et murins, en particulier au niveau du site catalytique, peut suggérer que ces protéines sont fonctionnelles et que leurs substrats sont proches de ceux qui sont connus chez l'homme et la souris.

La collagénase 3 humaine clive les collagènes interstitiels avec une préférence pour le collagène de type II [13]. On peut souligner que le collagène de type II est un composant majeur de la matrice extracellulaire des cartilages et que, dans la tête du xénope au cours de la métamorphose, la collagénase 3 est exprimée dans les cartilages qui se résorbent. La ST3 humaine ne clive aucun composant majeur de la matrice extracellulaire contrairement à la ST3 de souris qui clive la fibronectine et le collagène de type IV. L'incapacité de la ST3 humaine à cliver ces substrats est due à la présence, en position 235, d'une alanine à la place d'une proline [14]. Comme la ST3 de xénope possède une proline à la position correspondante, on peut penser que ses substrats sont les mêmes que ceux de la ST3 murine [8]. La fibronectine et le collagène de type IV sont en outre les composants majeurs de la lame basale, et la ST3, au cours de la métamorphose, est toujours exprimée dans des fibroblastes à proximité des lames basales. Les hypothèses de substrats pour la Col3 et la ST3 sont ainsi soutenues par les sites d'expression de leurs gènes au cours de la métamorphose du xénope.

Ainsi, non seulement le contrôle de l'expression de ST3, Col 3 et Col4 est différent, mais leurs substrats sont aussi probablement différents. Il est alors possible d'imaginer qu'elles agissent de concert pour permettre les modifications des différentes matrices extracellulaires nécessaires au déroulement de la métamorphose.

L'expression des MMP est donc finement contrôlée, notamment par les hormones thyroïdiennes. En outre, ces enzymes sont présentes aux endroits précis où elles doivent exercer une action spécifique. Cepen- dant, existe-t-il des preuves claires de leur rôle dans les remaniements tissulaires qui se produisent au cours de la métamorphose? Pour l'instant, seule la fonction de la ST3 a été étudiée pendant la métamorphose du xénope, et ce dans l'intestin par la méthode de culture d'explants [15]. L'utilisation d'un anticorps dirigé contre le domaine catalytique de la ST3 a permis de bloquer son activité et d'en étudier les conséquences. Quand cet anticorps est ajouté au milieu de culture d'intestin, on observe, en présence d'hormones thyroïdiennes, un blocage de l'apoptose des cellules épithéliales larvaires, et une inhibition ou un retard de remodelage de la matrice extracellulaire. Il apparaît donc que la ST3 joue un rôle, de façon directe ou indirecte, dans l'apoptose des cellules épithéliales larvaires et dans le remodelage de la lame basale qui lui est associée. Il serait intéressant que le même type d'expériences soit mené dans les autres organes dans lesquels le gène de la ST3 est activé au cours de la métamorphose.

\section{Et chez les mammifères ?}

Les MMP semblent donc jouer un rôle fondamental dans le remodelage des différentes matrices extracellulaires, permettant ainsi les changements tissulaires variés observés au cours de la métamorphose des amphibiens. Il reste encore beaucoup de recherches à effectuer pour mieux comprendre le contrôle de l'activité de ces enzymes, et leur fonction dans les différents organes. Toutes ces données obtenues chez les amphibiens pourraient permettre de préciser, chez les mammifères, la fonction des MMP et du contrôle de leur expression par les hormones thyroïdiennes non seulement pendant le développement normal, mais aussi au cours des processus pathologiques faisant intervenir ces protéines. Une telle approche comparative des différents modèles pourrait se révéler très fructueuse. Par la qualité de l'approche génétique qu'ils autorisent, les mammifères, et bien sûr en premier lieu la souris, rendent possible l'obtention de données très précises sur la réponse aux hormones thyroïdiennes et les liens unissant MMP, remodelage tissulaire et action hormonale. Ainsi, l'utilisation de la technique de microarray a permis de déterminer récemment les différents types de gènes contrôlés par les hormones thyroïdiennes dans le foie de la souris [16]. Inversement, en mettant à notre disposition un phénomène fascinant, complexe, mais puissamment ordonné, les amphibiens, et notamment le xénope, permettent de mieux comprendre la logique existant dans les cascades d'expression géniques. Les méthodes de transgenèse sont maintenant applicables au xénope, ce qui permettra de tester in vivo la réalité de la cascade de contrôle génique par la T3 au cours de la métamorphose. Un autre intérêt de cette approche comparative est de mieux comprendre comment les changements des contrôles moléculaires du développement peuvent jouer un rôle dans les modifications des processus de développement qui ont lieu au cours de l'évolution des organismes.

\section{Remerciements}

Nous remercions le CNRS, le MENRT et notamment le programme des ACI, ainsi que l'ARC et la région Rhône-Alpes pour leur soutien financier. Merci également à Catherine Hänni, Oriane Marchand, Rachid Safi et Michelina Plateroti pour la relecture de ce manuscrit.

\section{Stéphanie Bertrand Vincent Laudet}

Laboratoire de biologie moléculaire et cellulaire, Cnrs UMR 5665, École Normale Supérieure de Lyon, 46, allée d'Italie, 69364 Lyon Cedex 07, France.

\section{TIRÉS À PART}

V. Laudet. 


\section{RÉFÉRENCES}

1. Brown DD, Wang Z, Furlow JD, et al. The thyroid hormone-induced tail resorption program during Xenopus laevis metamorphosis. Proc Natl Acad Sci USA 1996; 93 : 1924-9

2. Wang Z, Brown DD. A gene expression screen. Proc Natl Acad Sci USA, 1991; 88: 11505-9.

3. Chassande O, Flamant F, Samarut J. Thyroid hormone receptor knock out: their contribution to our understanding of thyroid hormone resistance. Curr Opin Endocrinol Diabetes 1999; 6 : 293-300.

4. Murata E, Akita M, Kaneko K, Merker HJ. Changes associated with the basal lamina during metamorphosis of Xenopus laevis. Acta Anat 1994; 150 : 178-85.

5. Elinson RP, Remo B, Brown DD. Novel structural elements identified during tail resorption in Xenopus laevis metamorphosis : lessons from tailed frogs. Dev Biol 1999; $215: 243-52$
6. Gross J. How tadpoles lose their tails. $J$ Invest Dermatol 1966; 47: 274-7.

7. Stolow MA, Bauzon DD, Li J, et al. Identification and characterization of a novel collagenase in Xenopus laevis: possible roles during frog development. Mol Biol Cell $1996 ; 7: 1471-83$.

8. Patterton D, Hayes WP, Shi YB. Transcriptional activation of the matrix metalloproteinase gene stromelysin-3 coincides with thyroid hormone-induced cell death during frog metamorphosis. Dev Biol 1995; $167:$ 252-62.

9. Berry DL, Schwartzman RA, Brown DD. The expression pattern of thyroid hormone response genes in the tadpole tail identifies multiple resorption programs. Dev Biol 1998; 203: 12-23.

10. Wang Z, Brown DD. Thyroid hormoneinduced gene expression program for amphibian tail resorption. J Biol Chem 1993; 268: 16270-8.

11. Li J, Liang VC, Sedgwick T, Wong J, Shi YB. Unique organization and involvement of GAGA factors in transcriptional regula- tion of the Xenopus stromelysin-3 gene. Nucleic Acids Res 1998; 26 : 3018-25.

12. Ludwig MG, Basset P, Anglard P. Multiple regulatory elements in the murine stromelysin-3 promoter. J Biol Chem 2000; 275: 39981-90.

13. Knauper V, Lopez-Otin C, Smith B, Knight G, Murphy G. Biochemical characterization of human collagenase-3. J Biol Chem $1996 ; 271: 544-50$.

14. Noel A, Santavicca M, Stoll I, et al. Identification of structural determinants controlling human and mouse stromelysin-3 proteolytic activities. I Biol Chem 1995; 270 : 22866-72.

15. Ishizuya-Oka A, Li Q, Amano T, Damjanovski S, Ueda S, Shi YB. Requirement for matrix metalloproteinase stromelysin-3 in cell migration and apoptosis during tissue remodeling in Xenopus laevis. I Cell Biol $2000 ; 150: 1177-88$.

16. Feng X, Jiang Y, Meltzer P, Yen PM. Thyroid hormone regulation of hepatic genes in vivo detected by complementary DNA microarray. Mol Endocrinol 2000; 14: 947-55.

\section{口 BRÈVES}

L'IL-18: un nouvel acteur de l'athérosclérose. Il est maintenant admis que l'athérosclérose est une maladie inflammatoire chronique des artères qui a pour origine une agression initiale par le cholestérolLDL sous forme oxydée, et dans laquelle de nombreux médiateurs pro-inflammatoires sont clairement impliqués [1, 2]. Récemment, Z. Mallat dans le groupe d'A. Tedgui a montré que l'IL-18, une cytokine pro-inflammatoire de la famille de l'IL-1 $\beta$, est exprimée dans les plaques d'athérosclérose, en particulier quand il existe des signes d'instabilité [3]. Produite par les macrophages, l'IL-18 stimule, en synergie avec l'IL-12, la production par les lymphocytes Th1 d'interféron $\gamma$, un des facteurs pro-inflammatoires clés impliqués dans la pathogénie de l'athérosclérose. Elle induit aussi l'expression par les cellules endothéliales de molécules d'adhérence et de nombreuses chi- miokines. Tous ces éléments évoquaient un rôle de l'IL-18 dans l'athérosclérose. On sait en outre que l'activité de l'IL-18 est bloquée par un inhibiteur endogène appelé l'IL-18BP (pour IL-18 binding protein). La même équipe a donc évalué in vivo les effets de l'administration d'IL-18BP (par électrotranfert intramusculaire d'un plasmide exprimant l'IL-18BP) dans un modèle murin bien connu d'athérosclérose, les souris déficientes pour le gène de l'apoliproprotéine $\mathrm{E}$ [3] L'âge de début du traitement (14 semaines) a été judicieusement choisi afin d'étudier à la fois le développement de nouvelles plaques (dans l'aorte thoracique descendante) et l'évolution des plaques déjà constituées (dans le sinus aortique). L'effet protecteur de l'IL-18BP est très net, aussi bien sur le développement de nouvelles lésions que sur les plaques déjà constituées. Celles-ci sont profondé- ment modifiées avec une réduction de l'infiltration macrophagique, de l'apoptose et du contenu lipidique, et une augmentation du nombre de cellules musculaires lisses et du contenu en collagène. Tous ces effets sont connus pour favoriser la stabilité des plaques d'athérosclérose et donc limiter la survenue de rupture, érosion ou thrombus. L'IL18 est donc à ajouter à la liste des cytokines inflammatoires impliquées dans l'athérosclérose et dont le blocage des voies de signalisation représente une voie thérapeutique potentielle.

[1. Tedgui, et al. Med Sci 2001; 17 : 162-9.]

[2. Bonnet J. Med Sci 2001 ; 17 : 55967.]

[3. Mallat Z, et al. Circulation 2001; 104: 1598-603.]

[4. Mallat Z, et al. Circ Res 2001; 89: e41-5.] 
